\title{
Design of superhydrophobic strain sensor with a multi-layer structure for human motion monitoring
}

Wei-Chen Gao ${ }^{a \#, ~ W e i ~ W u ~}{ }^{b \#}$, Chang-Zhou Chen ${ }^{a}$, Hui Zhao ${ }^{a, c, d, e^{*}}$, Yang Liu ${ }^{a}$, Qing Li ${ }^{a, d}$, Chong-Xing Huang ${ }^{a}$, Guo-hua Huf, Shuang-Fei Wang ${ }^{a}$, Dean Shic, Qun-Chao Zhang ${ }^{c}$

a. Guangxi Key Laboratory of Clean Pulp \& Papermaking and Pollution Control, School of Light Industry and Food Engineering, Guangxi University, Nanning, 530004, China.

b. Department of Materials Science and Engineering, City University of Hong Kong, Tat Chee Avenue, Kowloon, Hong Kong SAR, China.

c. State Key Laboratory of Biocatalysis and Enzyme Engineering, School of Life Sciences, Key Laboratory for the Green Preparation and Application of Functional Materials, Hubei University, Wuhan 430062, China.

d. Guangxi Key Laboratory of Chemistry and Engineering of Forest Products, Guangxi Collaborative Innovation Center for Chemistry and Engineering of Forest Products, Guangxi University for Nationalities, Nanning 530006, China.

e. Guangxi Bossco Environm Protect Technol Co Ltd, Nanning, Guangxi, China.

f. University of Lorraine - CNRS, Laboratory of Reactions and Process Engineering (LRGP, UMR 7274), ENSIC, 1 rue Grandville, BP 20451, 54001 Nancy Cedex, France. 


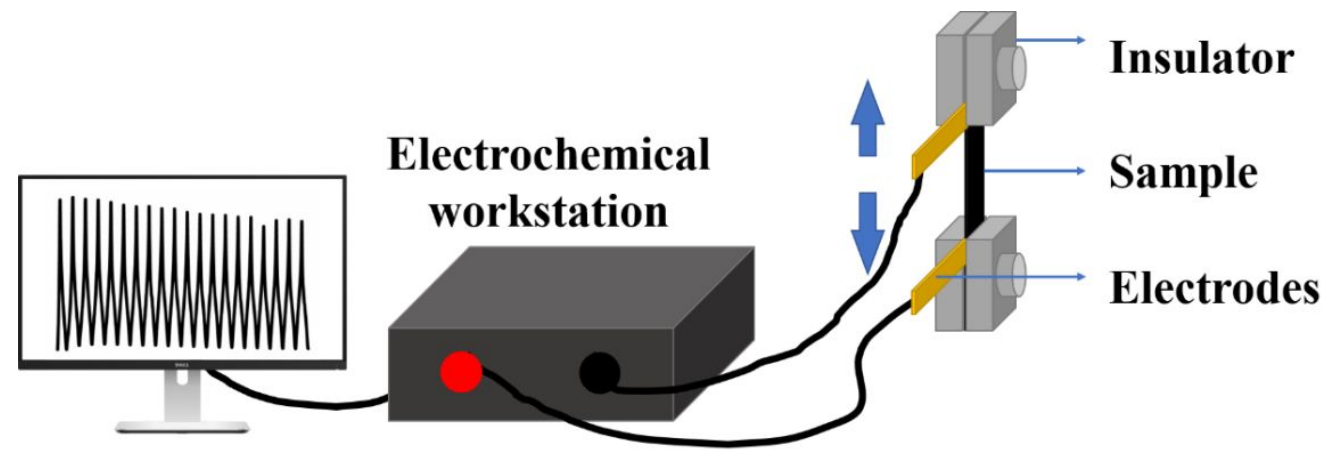

Figure S1. Sensor performance test diagram.

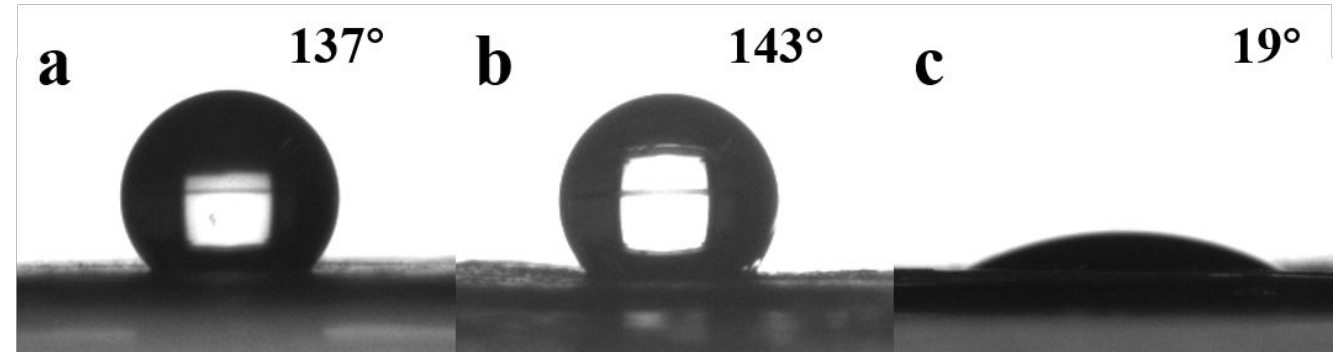

Figure S2. WCA: (a)PU/PDA/PFDT, (b)PU/RGO/PFDT, (c)PU/PDA.
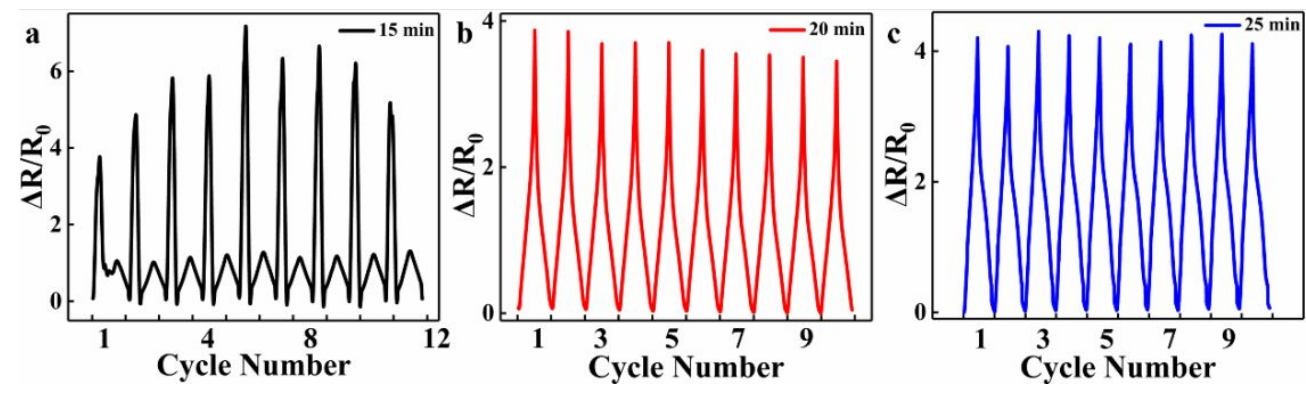

Figure S3. Effect of ultrasonic treatment time on the electrical properties of materials.
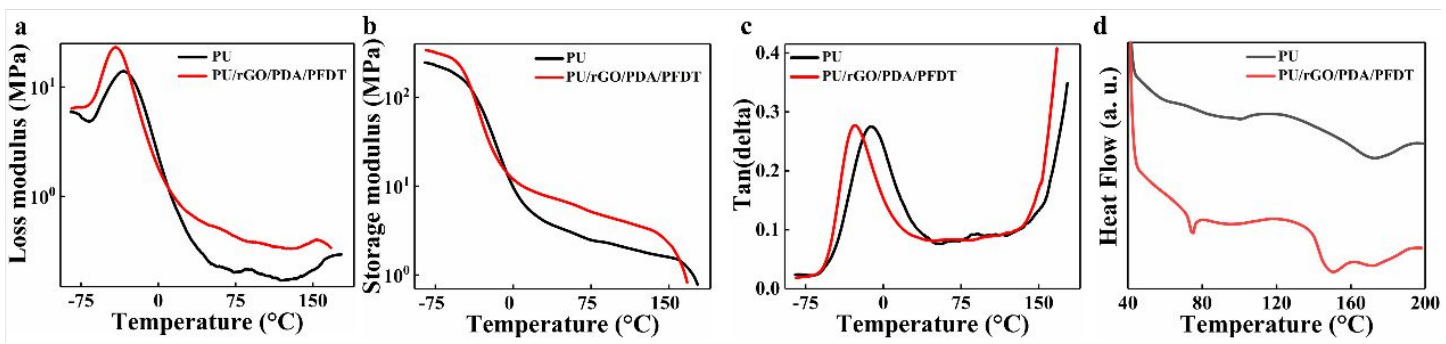

Figure S4. (a-c) is the DMA test result, (d) DSC curve of fiber film. 


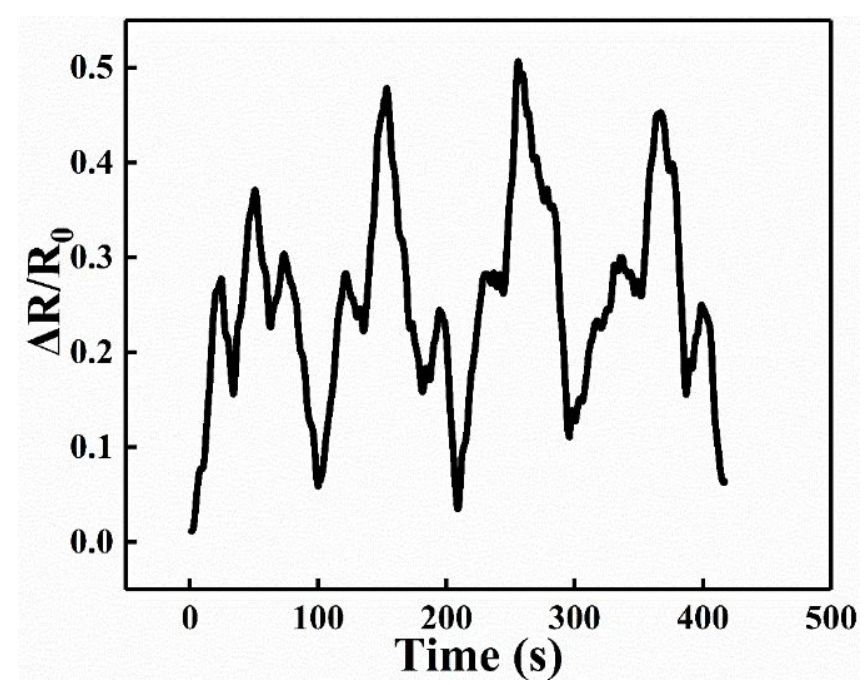

Figure S5. $R_{0^{-}}$curve of material not coated with PDA.

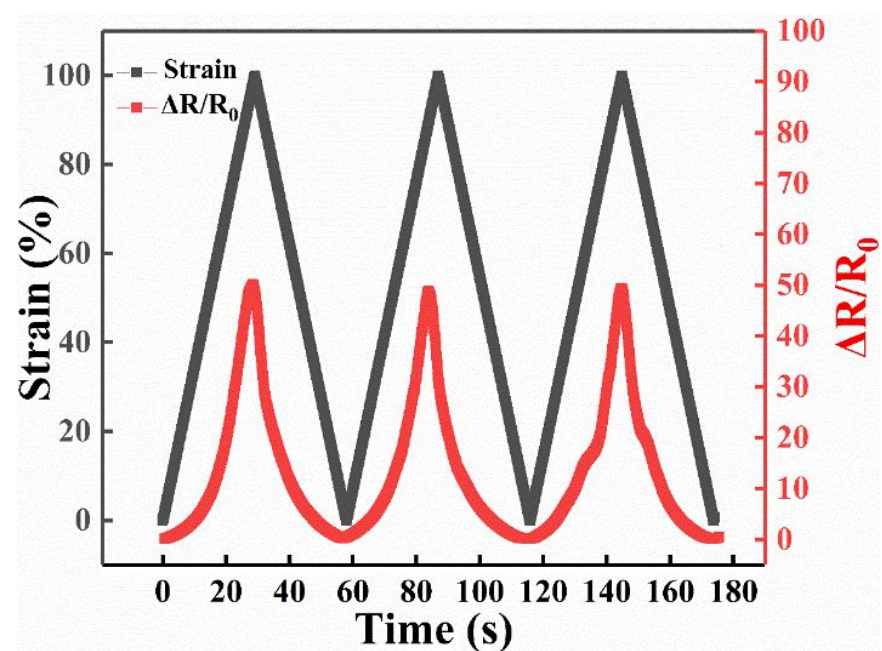

Figure S6. The matching relation between $\Delta R / R_{0}$ and displacement.

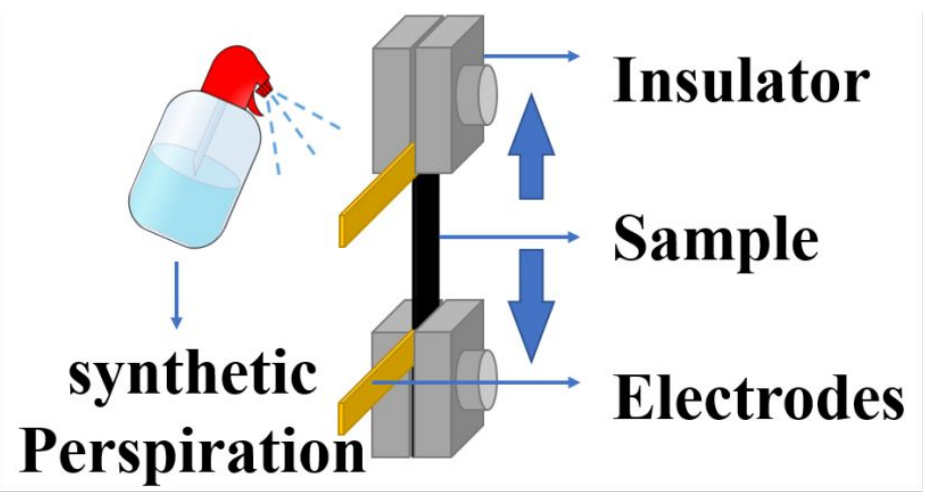

Figure S7. Schematic diagram of electrical performance test of sensor in high humidity environment. 


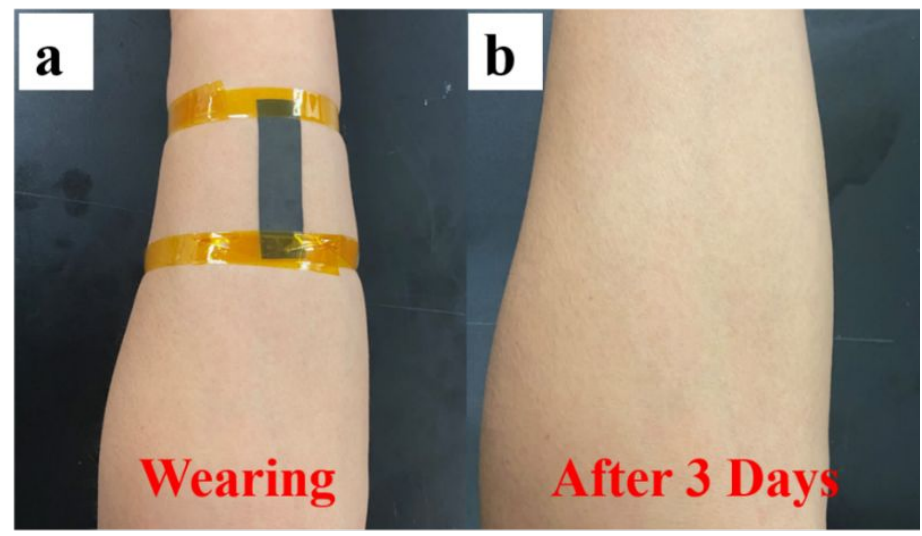

Figure S8. Authors wore a sensor on his arm for three days.

Table S1 A comparison of work in recent years.

\begin{tabular}{ccccc}
\hline Conductive fillers & Polymer materials & Strain (\%) & GF & Reference \\
\hline \multirow{2}{*}{ Silver nanowires } & PU & 400 & 1 & 10 \\
& PDMS & 50 & 12.5 & 15 \\
\hline ZnO & PU & 150 & 15 & 11 \\
Carbon & Cellulose & 140 & 25 & 14 \\
Graphite & Protein & 30 & 14 & 18 \\
\hline \multirow{2}{*}{ RGO } & PU & 600 & 79 & 12 \\
& PDMS & 60 & 4 & 16 \\
& PU & 400 & 5.9 & 20 \\
\hline & Rubber & 400 & 3.3 & 5 \\
CNT & PU/SEBS & 300 & 2.8 & 9 \\
& PU & 100 & 110 & 13 \\
& Polyamide 6 & 40 & 1.9 & 17 \\
\hline RGO & PU & 590 & 221 & This work \\
\hline
\end{tabular}

Table S2 Water vapor permeability detection.

\begin{tabular}{ccc} 
Sample name & $\begin{array}{c}\text { Water vapor transmittance } \\
\left(\mathrm{g} /\left(\mathrm{m}^{2 *} 24 \mathrm{~h}\right)\right)\end{array}$ & $\begin{array}{c}\text { Water vapor transmission coefficient } \\
\left(\mathrm{g} * \mathrm{~cm} /\left(\mathrm{cm}^{2 *} \mathrm{~s} * \mathrm{~Pa}\right)\right)\end{array}$ \\
\hline PU & 2339.45 & $3.992 * 10^{-12}$ \\
$\mathrm{PU} / \mathrm{RGO} / \mathrm{PDA} / \mathrm{PFDT}$ & 2348.89 & $3.862 * 10^{-12}$ \\
\hline
\end{tabular}

